\title{
Thermodynamics of the Adsorption of Flexible Polymers on Nanowires
}

\author{
Thomas Vogel, , , J Jonathan Gross, ${ }^{2,3, \text {, }}$ and Michael Bachmann ${ }^{3,4,5}$, 团 \\ ${ }^{1}$ Theoretical Division (T-1), Los Alamos National Laboratory, Los Alamos, NM 87545, USA \\ ${ }^{2}$ Institut für Theoretische Physik and Centre for Theoretical Sciences (NTZ), \\ Universität Leipzig, Postfach 100 920, D-04009 Leipzig, Germany \\ ${ }^{3}$ Soft Matter Systems Research Group, Center for Simulational Physics, \\ The University of Georgia, Athens, GA 30602, USA \\ ${ }^{4}$ Instituto de Física, Universidade Federal de Mato Grosso, 78060-900 Cuiabá (MT), Brazil \\ ${ }^{5}$ Departamento de Física, Universidade Federal de Minas Gerais, 31270-901 Belo Horizonte (MG), Brazil
}

(Dated: June 20, 2021)

\begin{abstract}
Generalized-ensemble simulations enable the study of complex adsorption scenarios of a coarsegrained model polymer near an attractive nanostring, representing an ultrathin nanowire. We perform canonical and microcanonical statistical analyses to investigate structural transitions of the polymer and discuss their dependence on the temperature and on model parameters such as effective wire thickness and attraction strength. The result is a complete hyperphase diagram of the polymer phases, whose locations and stability are influenced by the effective material properties of the nanowire and the strength of the thermal fluctuations. Major structural polymer phases in the adsorbed state include compact droplets attached to or wrapping around the wire, and tubelike conformations with triangular pattern that resemble ideal boron nanotubes. The classification of the transitions is performed by microcanonical inflection-point analysis.
\end{abstract}

\section{INTRODUCTION}

Although the recent advances in nanotechnology make it possible to study smaller systems than ever before, the systematic understanding of miniature structures of macromolecules attached to inorganic adsorbents is an experimental challenge [1, 2]. The typical approaches of experimental verification of theoretical predictions and the theoretical understanding of experimental results is often limited to very specific questions [3] . On the other hand, computer simulations are a comparatively inexpensive tool that allows for comprising studies, which can address generic problems as well. Pertaining to polymer adsorption at substrates, generic properties of this transition include common transition pathways from the desorbed into the different adsorption phases or vice versa ("wetting", "dewetting"), but also transitions between structural phases under the influence of external factors such as temperature and solvent quality. General material properties of the adsorbent, e.g., the propensity to bind macromolecules and the attraction (or repulsion) range, affect the binding process as well.

There have been numerous computational studies of this complex behavior by means of simplified, coarsegrained models in the recent past. Yet, there are still more questions than answers about the nature of the adsorption phases that are difficult to approach. Strictly, most of these structural phases are not phases in the thermodynamic sense, since the features of these phases and the transitions in between them are governed or at least substantially influenced by finite-size effects [4]. It has

\footnotetext{
* Contact: tvogel@lanl.gov http://www.thomasvogel.name

$\dagger$ E-mail: jonathan.gross@itp.uni-leipzig.de

‡ E-mail: bachmann@smsyslab.org

Homepage: http://www.smsyslab.org
}

been a fairly profound finding that the thermodynamic behavior of compact phases of finite polymers is rather irregular due to discrete crystal symmetries and surface effects [5 7].

Most of the recent generic studies aiming at unraveling the structure of the phase space and the adsorption dynamics have been performed for polymers and proteins interacting with a perfectly flat substrate with [8 10] or without pattern [11 23]. Much less literature exists about polymer adsorption at fluctuating surfaces such as membranes [24, 25], because of the substantially increased complexity of the problem. Flexible polymers can also optimally adapt to curved surfaces such as cylindrical substrates 26 28], which causes the formation of polymer structures different from the adsorption phases at flat substrates.

Nanotube-polymer composites are a particularly interesting class of hybrid systems with potential for nanotechnological applications. Besides the individual mechanical and electronic properties of nanotubes, in particular carbon nanotubes [29, 30], hybrid nanotubepolymer systems have an even broader spectrum of advanced applications in technology and medicine. These features have been exploited, for example, in photonics 31] and nanotubes coated with conducting polymers were used to design biosensors [32]. Potential medical applications include serum protein-coated gold nano-rods for the selective targeting of cancer cells [33, 34]. The understanding of the polymer wetting behavior of nanotubes [35] is a key to further developments in the technological preparation of such hybrid systems. The investigation of the interaction of macromolecules with carbon nanotubes has also been the subject of numerous computational studies 36 -42].

Recently, we investigated the extreme case of polymers adsorbed on ultrathin nanowires and constructed 
a structural phase diagram based on stable low-energy states [43]. This is particularly interesting, because the nanowire geometry is topologically different from a nanocylinder, which leads to a different monomersubstrate interaction model. Therefore, to consider the nanowire as the limiting case of a nanocylinder with zero radius is nontrivial. Experimentally resolved structures, for example for the spread of glycerol droplets on carbon fibers and epoxy resin on aramid filaments [44] or liquid droplets at thin cylinders [45], exhibit similarities to the adsorbed, globular conformations we found in our simulations of a simple coarse-grained polymernanowire model. The outer membrane of certain bacteria possess an ordered, hexagonally packed surface layer and although the units of the outer protein layer are not formed by polymers, images of long tubular portions surrounded by hexagonally packed units [46] resemble polymer-tube structures we identified for strongly attractive nanowires [43]. Potential applications of hybrid polymer-nanowire assemblies include tube systems for the transport of small molecules that can basically be modeled in any desired shape.

In this paper, we extend our previous study of properties of lowest-energy polymer adsorption phases [43] by introducing the temperature as the external control parameter for thermal fluctuations. By means of generalized-ensemble Monte Carlo methods and by systematic variation of temperature and model parameters such as effective wire thickness (volume exclusion) and attraction strength, we investigate the thermodynamic properties of the structural phases of adsorbed and desorbed polymers and the transitions between them. The competition between energetic ordering and entropic diversity that causes structural transitions will be investigated by the conventional canonical statistical analysis of thermodynamic response quantities and more advanced approaches such as the microcanonical inflection-point analysis [4, 47]. The latter method has proven to be particularly useful, whenever finite-size effects dominate or substantially influence structural transitions.

The paper is organized as follows. After the introduction of the hybrid polymer-wire model in Sect. [I] we briefly review the conformational phase diagram of polymer ground-state structures adsorbed to a nanowire in Sect. III. Results for the adsorption thermodynamics of the polymer are analyzed and discussed in Sect. IV] The microcanonical analysis of the adsorption transition is performed in Sect. V] Our main conclusions are summarized in Sect. VI

\section{THE POLYMER-WIRE MODEL}

We employ a generic, coarse-grained model for a selfinteracting homopolymer with $N$ monomers in the proximity of an attractive one-dimensional stringlike substrate. The energy of a conformation $\mathbf{X}$ of the polymer consists of contributions for the interaction between nonbonded monomers, bending stiffness of the polymer chain, and the interaction of each monomer with the nanowire:

$$
\begin{aligned}
E(\mathbf{X})= & \sum_{i=1}^{N-2} \sum_{j=i+2}^{N} V_{\mathrm{LJ}}\left(r_{i j}\right)+\sum_{i=2}^{N-1} V_{\text {bend }}\left(\cos \theta_{i}\right) \\
& +\sum_{i=1}^{N} V_{\text {string }}\left(r_{\perp ; i}\right) .
\end{aligned}
$$

The pairwise interaction between nonbonded monomers is described by a standard Lennard-Jones potential,

$$
V_{\mathrm{LJ}}\left(r_{i j}\right)=4 \epsilon_{\mathrm{m}}\left[\left(\frac{\sigma_{\mathrm{m}}}{r_{i j}}\right)^{12}-\left(\frac{\sigma_{\mathrm{m}}}{r_{i j}}\right)^{6}\right],
$$

where $r_{i j}$ is the distance between monomers $i$ and $j$. The potential minimum $V_{\mathrm{LJ}}\left(r_{i j}^{\min }\right)=-\epsilon_{\mathrm{m}}$ is located at $r_{i j}^{\min }=2^{1 / 6} \sigma_{\mathrm{m}}$. In our simulations, we used energy and length units for which $\epsilon_{\mathrm{m}}=1$ and $\sigma_{\mathrm{m}}=1$, respectively. Bonds between monomers adjacent along the chain are stiff and of unit length, $r_{i i+1}=1$. Covalent bond vectors connected to the $i$ th monomer form a bending angle $\theta_{i}$. The bending energy

$$
V_{\text {bend }}\left(\cos \theta_{i}\right)=\kappa\left(1-\cos \theta_{i}\right)
$$

is a remnant of the $\mathrm{AB}$ model, which has initially been introduced as a coarse-grained hydrophobic-polar peptide model [48] that has proven to be useful for the qualitative description of generic features of conformational transitions associated with protein folding [49] and aggregation processes [50]. In this model, the bending stiffness is set to $\kappa=1 / 4$. The homopolymer version of the model has also been used in recent adsorption studies of polymers [10, 18, 22, 43, 51].

For the determination of the string potential, we consider a monomer interacting with each infinitesimal element of the string, which is supposed to extend infinitely into $\pm z$ direction. We assume that this interaction is of van der Waals type and can be described by a LennardJones potential. By using the cylindrical coordinate representation, the total potential of a monomer $i$ in the field of the string is then given by [43, [51]

$$
\begin{aligned}
V_{\text {string }}\left(r_{\perp ; i}\right) & =4 \eta_{\mathrm{f}} \epsilon_{\mathrm{f}} \int_{-\infty}^{\infty} \mathrm{d} z\left[\frac{\sigma_{\mathrm{f}}^{12}}{\left(r_{\perp ; i}^{2}+z^{2}\right)^{6}}-\frac{\sigma_{\mathrm{f}}^{6}}{\left(r_{\perp ; i}^{2}+z^{2}\right)^{3}}\right] \\
& =\pi \eta_{\mathrm{f}} \epsilon_{\mathrm{f}}\left(\frac{63}{64} \frac{\sigma_{\mathrm{f}}^{12}}{r_{\perp ; i}^{11}}-\frac{3}{2} \frac{\sigma_{\mathrm{f}}^{6}}{r_{\perp ; i}^{5}}\right),
\end{aligned}
$$

where we have introduced the perpendicular distance $r_{\perp ; i}$ of the monomer from the string axis and the van der Waals radius or effective "thickness" of the string $\sigma_{\mathrm{f}}$, which is related to the potential minimum distance $r_{\perp}^{\mathrm{min}}$ via:

$$
r_{\perp}^{\min }=\left(\frac{693}{480}\right)^{1 / 6} \sigma_{\mathrm{f}} \approx 1.06 \sigma_{\mathrm{f}}
$$




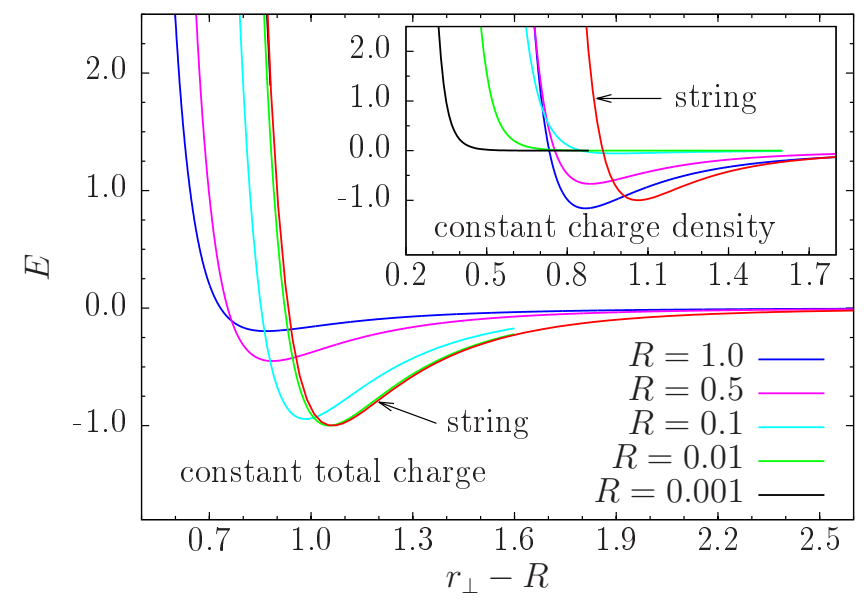

FIG. 1. Cylinder potential [Eq. [6] ] for different radii $R \in$ $(0 \ldots 1]$ and the string potential [Eq. (4)] for $\sigma_{\mathrm{f}}=\epsilon_{\mathrm{f}}=1$ for constant total "charge", i.e., $\eta_{\text {cyl }}=1 / \pi R^{2}$ (main figure) and for constant "charge" density $\eta_{\mathrm{cyl}}=1$ (inset). The inset figure corresponds to Fig. 4 in Ref. [26].

The string "charge" density $\eta_{\mathrm{f}}$ compensates for the additional dimension by the extra $\sigma_{\mathrm{f}}$ factor that remains after integration over the standard form of the Lennard-Jones potential. It can be conveniently used to set the energy scale of the potential. We adjust it in such a way that the minimum monomer-string energy is $V_{\text {string }}\left(r_{\perp}^{\min }\right)=-\epsilon_{\mathrm{f}}$, independently of $\sigma_{\mathrm{f}}$. In this case, $\eta_{\mathrm{f}} \approx 0.528 / \sigma_{\mathrm{f}}$.

It is instructive to view the string potential (4) from a different perspective, namely as the $R \rightarrow 0$ limit of the corresponding potential of an attractive cylinder. This is nontrivial, because of the different topological dimensions of string and cylinder. The cylinder-monomer potential can be written as [26]:

$$
\begin{aligned}
& V_{\text {cyl }}(R,\left.r_{\perp ; i}\right)=\pi \epsilon_{\mathrm{f}} \eta_{\text {cyl }} \int_{0}^{2 \pi} \mathrm{d} \phi \int_{0}^{R} \rho \mathrm{d} \rho \\
& \times\left(\frac{63}{64} \frac{\sigma_{\mathrm{f}}^{12}}{r^{\prime 11}\left(r_{\perp ; i}, \rho, \phi\right)}-\frac{3}{2} \frac{\sigma_{\mathrm{f}}^{6}}{r^{\prime 5}\left(r_{\perp ; i}, \rho, \phi\right)}\right),
\end{aligned}
$$

where the integration in $z$ direction has already been performed. The distance between the $i$ th monomer and any point in the cylinder disk in-plane with the monomer is given by $r^{\prime}=\left(r_{\perp ; i}^{2}+\rho^{2}-2 \rho r_{\perp ; i} \cos \phi\right)^{1 / 2} ; \rho$ and $\phi$ are the polar coordinates in the plane of cylinder disk and monomer. Compared to Ref. 26], we have introduced in the potential (6) the volume "charge" density $\eta_{\text {cyl }}$ of the cylinder. If this density is considered a constant and is thus independent of $R$ (as in Ref. [26]), the string potential (4) cannot be obtained as the $R \rightarrow 0$ limit of the cylinder potential. Only if the total "charge" is fixed, i.e., $\eta_{\text {cyl }}$ scales with the inverse area of the cylinder disk $1 / \pi R^{2}$, then

$$
V_{\text {string }}\left(r_{\perp ; i}\right)=\lim _{R \rightarrow 0} V_{\text {cyl }}\left(R, r_{\perp ; i}\right) .
$$

For both cases, the $R \rightarrow 0$ limits of the cylinder potential are plotted in Fig. 1 The systematic discussion of polymer adsorption at cylindrical substrates will be done elsewhere [28].

In our model of the polymer-wire system, the polymer is not grafted to the string and can move freely. The string, pointing into $z$ direction, is located in the center of the periodic simulation box with edge lengths $2 N$ in $x$ and $y$ directions to prevent the polymer from escaping.

In our simulations, we performed different runs using multicanonical 52 54] and Wang-Landau sampling [55, 56] and found the results to be coherent. Conformational updates included local crankshaft and slithering-snake moves, as well as global spherical-cap [49] and translation moves [51]. The crankshaft move corresponds to the rotation of a single monomer about the axis given by the vector between its nearest neighbors and changes the conformation locally. For the slithering-snake update, a monomer is cut at one end of the chain and pasted at the other end, inverting the bond vector. This move proves useful to avoid trapping in very dense conformations. The spherical-cap update consists of a pivot rotation of a single bond and the subsequent shift of the monomers at either end of the polymer, keeping all bond lengths fixed. This update allows for larger steps in the conformational space. Finally, the global translation update allows for a displacement of the entire polymer chain relative to the string. Update types were chosen randomly with equal weight, which allowed for the efficient sampling of the conformational space.

The chain lengths studied varied from $N=30$ to $N=100$ for the analysis of the thermodynamic adsorption behavior and up to $N=200$ for the investigation of ground-state properties.

\section{LOWEST-ENERGY CONFORMATIONS OF ADSORBED POLYMERS}

The investigation of conformational properties of lowest-energy states is generally beneficial for the identification of compact crystalline or amorphous structural phases. The major ground-state morphologies of the polymer interacting with an attractive nanowire were found to be string-attached globular droplets and tubelike barrels wrapped around the string [43]. In the first case, depending on the attraction strength and effective thickness of the wire, the string penetrates the globule (Gi: globular, included) or it is only loosely attached to it (Ge: globular, excluded).

Most interesting is the formation of tubelike, or barrellike (B), polymer structures, which resemble nanotube morphologies [57]. This occurs if the string field is more attractive for most monomers than the compact crystalline assembly and the effectively excluded string diameter is small enough.

Topological transitions, common to all adsorption problems and typically accompanied by layering effects, can also be observed in the barrel phase. At very low $\sigma_{\mathrm{f}}$ values, where monomers are attached so close to the 

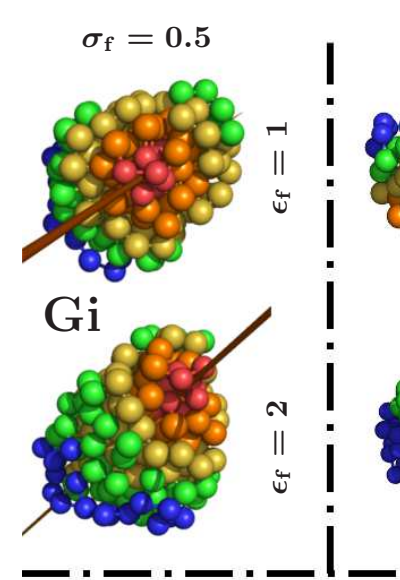

$\sigma_{\mathrm{f}}=1.5$

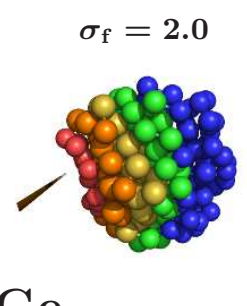

1
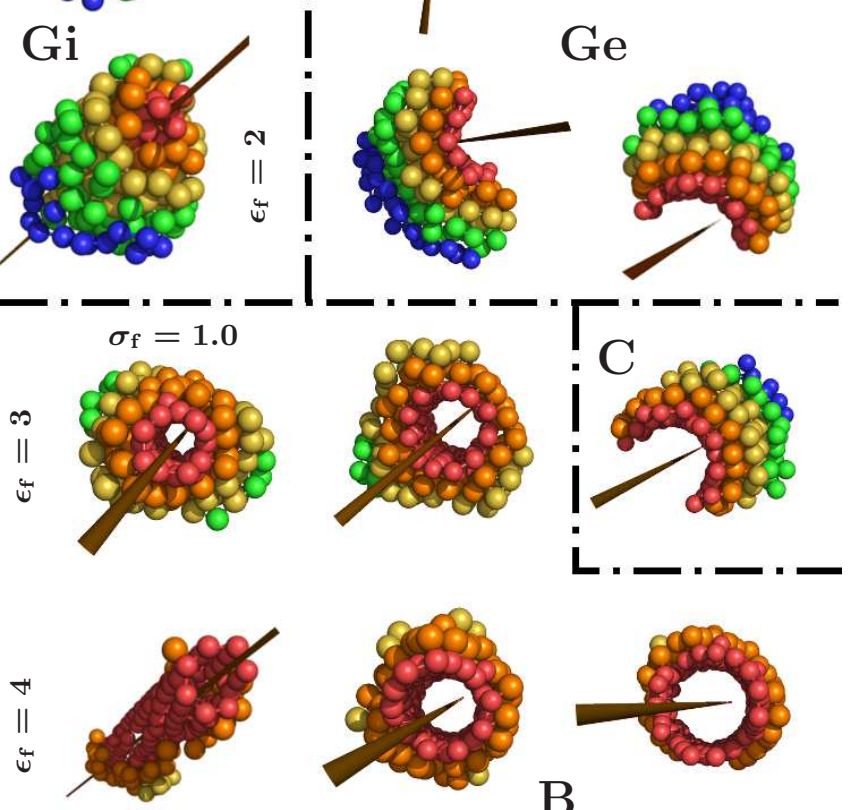

B
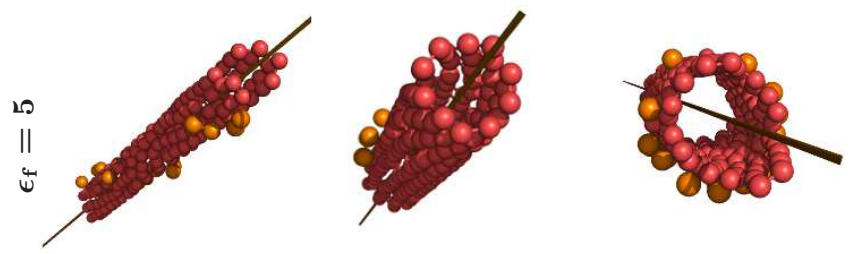

FIG. 2. Typical low-energy conformations of a polymer with $N=200$ monomers adsorbed at a string with effective thicknesses $\sigma_{\mathrm{f}}$-values 0.5 and 1 , respectively (first column), 1.5 (second) and 2 (third) for attraction strengths $\epsilon_{\mathrm{f}}=1,2,3,4$, and 5 (top to bottom). Monomers are marked with different shades according to their distance from the string.

string that they can feel the attractive field of monomers of the opposite side of the string as well, the polymer "tube" looks rather like a one-dimensional chain with zigzag (or sawtooth) pattern. In the other extreme case, if the effective string thickness is too large, additional monomers will attach to the two-dimensional monolayer tube, essentially forming a new layer. Three-dimensional assemblies with two or more layers are also known from atomic nanotubes [58].

For comparatively large string thickness and sufficiently high adsorption strength, the polymer wraps only partially around the string. This structural phase is very typical for polymer adsorption transitions at cylinders [26, 28] and has been called "clamshell" phase (C).

Figure 2 shows typical conformations in these different structural low-energy phases for a polymer with
200 monomers at various values of string parameters $\sigma_{\mathrm{f}}$ and $\epsilon_{\mathrm{f}}$, supporting our general findings for other chain lengths [43]. Since the monomer-string interaction is attractive, the polymer is adsorbed to the string in all low-energy phases.

In the following, we will investigate the influence of thermal fluctuations upon the the formation of compact adsorbed polymer structures and the thermodynamic properties of the adsorption transition.

\section{THERMODYNAMICS OF POLYMER ADSORPTION AT STRINGLIKE SUBSTRATES}

For the understanding of the thermodynamic behavior of the system, it is useful to perform canonical and microcanonical analyses of various thermodynamic quantities with the goal of identifying generic features of polymer adsorption at a stringlike nanowire for various parametrizations in the space of effective van der Waals radius $\sigma_{\mathrm{f}}$ of the wire and its attractive adsorption strength $\epsilon_{\mathrm{f}}$.

Figure 3 shows heat-capacity curves $C_{V}(T)$ for a polymer consisting of $N=30$ monomers interacting with various nanowires, distinguished by their different material parameters $\sigma_{\mathrm{f}}$ and $\epsilon_{\mathrm{f}}$. Most pronounced in all cases is the significant peak at high temperatures, which corresponds to the adsorption transition. For fixed values of $\sigma_{\mathrm{f}}$, the peak position, which we denote by $T_{\mathrm{ads}}^{\mathrm{can}}$ in the following, scales almost perfectly linearly with the wire attraction strength, $T_{\mathrm{ads}}^{\mathrm{can}} \propto \epsilon_{\mathrm{f}}$. This has already been found in adsorption studies at planar substrates [15, 18] and can intuitively be understood by the larger thermal energy $\sim k_{\mathrm{B}} T$ needed to release the polymer off the substrate at larger adsorption strengths. However, it is worth mentioning as well that the proportionality factor depends nonlinearly on the effective string thickness $\sigma_{\mathrm{f}}$.

This can also be seen in Fig. 4, where we have plotted the adsorption temperature as a function of $\sigma_{\mathrm{f}}$ and $\epsilon_{\mathrm{f}}$ for a 100-mer. We have also included the zero-temperature structural phase diagram from Ref. [43] into the $T=0$ plane to get an impression of how the compact adsorption phases at given $\sigma_{\mathrm{f}}$ and $\epsilon_{\mathrm{f}}$ values look like. Not surprisingly, the adsorption transition temperatures depend on $N$ and are different for the 100-mer, compared to the 30-mer. However, qualitatively the generic results are comparable.

After passing the adsorption transition, for temperatures $T_{\text {cryst }}^{\text {can }}<T<T_{\text {ads }}^{\text {can }}$, dominant polymer conformations are either disordered and expanded or locally ordered and globular. For the adsorption of the 30-mer at the nanowire, these phases are difficult to distinguish. More striking is the crystallization transition at $T_{\text {cryst }}^{\text {can }}$ into the compact adsorption phases that have been described qualitatively in Section III As it is obvious from Fig. 3. the low-temperature peak positions in the specific heat curves near $T_{\text {cryst }}^{\text {can }}$, indicating this transition, do not vary much and, therefore, hardly depend on the 


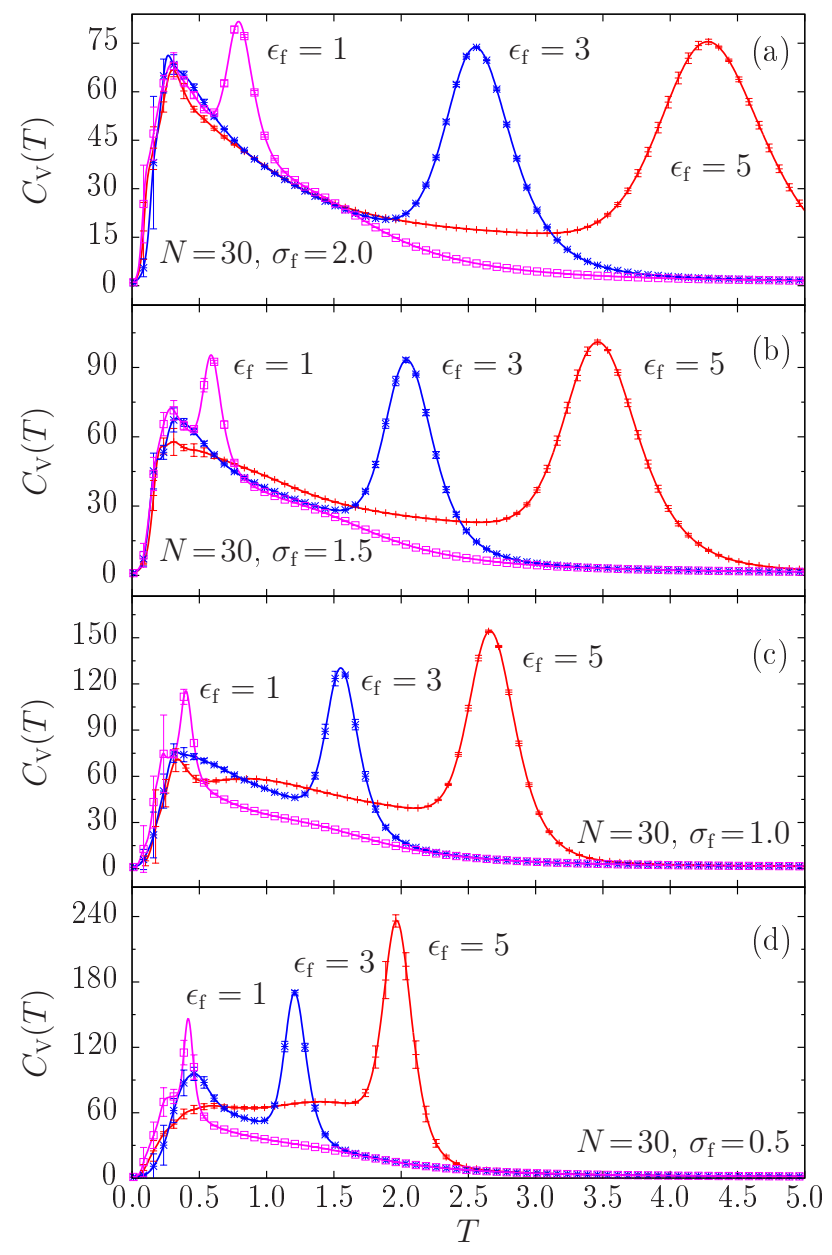

FIG. 3. Temperature dependence of the heat capacities of a 30-mer for different string attraction strengths $\left(\epsilon_{\mathrm{f}}=1,3\right.$, and 5, respectively) and decreasing van der Waals radii: (a) $\sigma_{\mathrm{f}}=2.0$, (b) 1.5, (c) 1.0, and (d) 0.5. Note the different ordinate scales. Error bars were estimated using the jackknife method [4].

material parameters of the nanowire, $\epsilon_{\mathrm{f}}$ and $\sigma_{\mathrm{f}}$. Effectively, during this transition, the number of contacts between monomers and wire does not change much and the way in which the polymer binds to the wire has already been established in the "liquid" (expanded or globular) phase. Note that, for strong attraction (large $\epsilon_{\mathrm{f}}$ values), the crystallization transition becomes weaker, the smaller the effective wire thickness is. This confirms the previous statement that the basic, tubelike structures have already formed in the "liquid" phase, with most monomers already in contact to the wire.

If the wire attraction and thickness are comparatively small $\left(\epsilon_{\mathrm{f}}<2\right.$ and $\left.\sigma_{\mathrm{f}}<1\right)$, the chain behaves basically like a free, flexible polymer, with minimal recognition of the wire, which is enclosed inside the polymer conformation for energetic reasons. In this case, the adsorbed liquid phase is preempted by the compact, crystalline phase. Adsorption and crystallization merge. Noteworthy are the very weak peaks in Fig. B at about $T_{\Theta}^{\text {can }} \approx 1.5$, which

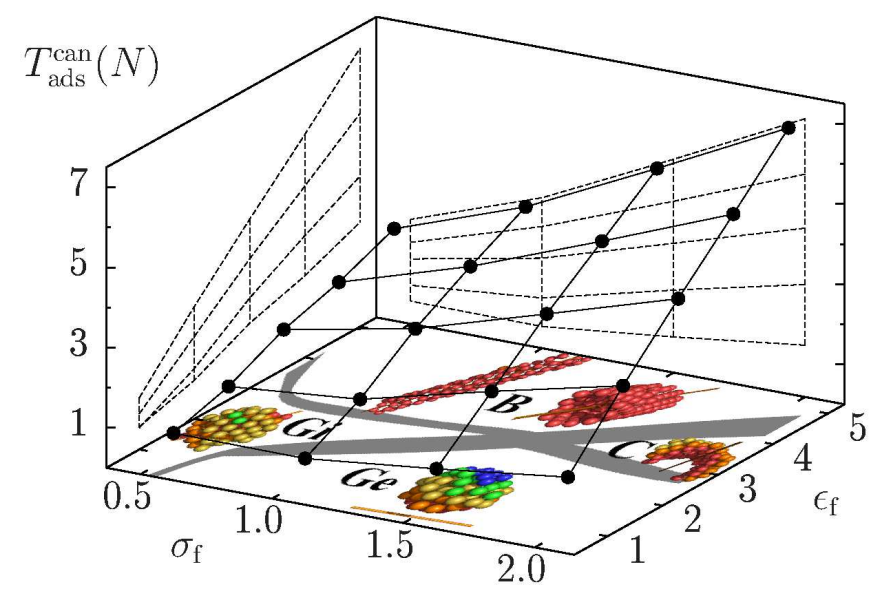

FIG. 4. Adsorption temperatures for different values of $\epsilon_{\mathrm{f}}$ and effective wire thickness $\sigma_{\mathrm{f}}$ for a polymer of length $N=100$. The structural phase diagram for ground-states from Ref. [43] is projected upon the $T=0$ plane.

indicate the $\Theta$ transition of the polymer chain. The weakness of the $\Theta$ transition for very short flexible and semiflexible chains has already been observed previously [5-7].

Figure 5 shows an example for a complete structural phase diagram at $\sigma_{\mathrm{f}}=1.0$ for $N=30$. Representative conformations in the different phases at parameter values indicated by the circles in the phase diagram are also depicted. The phase diagram prominently features the almost linear adsorption transition line between desorbed and adsorbed phases. Transitions from the desorbed extended (DE) and desorbed globular (DG) phases, which are separated by the well-known $\Theta$ transition line, into adsorbed phases lead to a variety of structures. However, given the rather small size of the polymer, these conformations can hardly be distinguished. Although we expect that the dominant DE structures will also adsorb as extended conformations and, similarly, DG structures cross over into adsorbed globules, a quantitative description is more difficult than in the adsorption scenarios at planar substrates [15, 18]. A significant signal for the separation of extended and globular in the adsorbed regime could not be identified. The "crystallization" transition into compact phases dominated by the ground-state structures, however, is clearly visible. In complete analogy to the previous analysis of ground-state structures for $\sigma_{\mathrm{f}}=1$ in the case of the 100-mer [43] (see also the $\sigma_{\mathrm{f}}=1$ cross section in the projection in Fig. (4), we clearly identify compact globular droplets with the string excluded $(\mathrm{Ge})$ at rather small values of the string attraction strength $\epsilon_{\mathrm{f}}$. With $\epsilon_{\mathrm{f}}$ increasing, the string becomes included into the globule (Gi). Only for sufficiently large string attraction $\left(\epsilon_{\mathrm{f}}>3\right)$, tubelike "barrel" (B) structures form. Further increasing $\epsilon_{\mathrm{f}}$, these become dominated by monolayer (or single-walled) polymer tubes. The detailed discussion of the ground-state dominated compact phases can be found in Ref. 43]. 

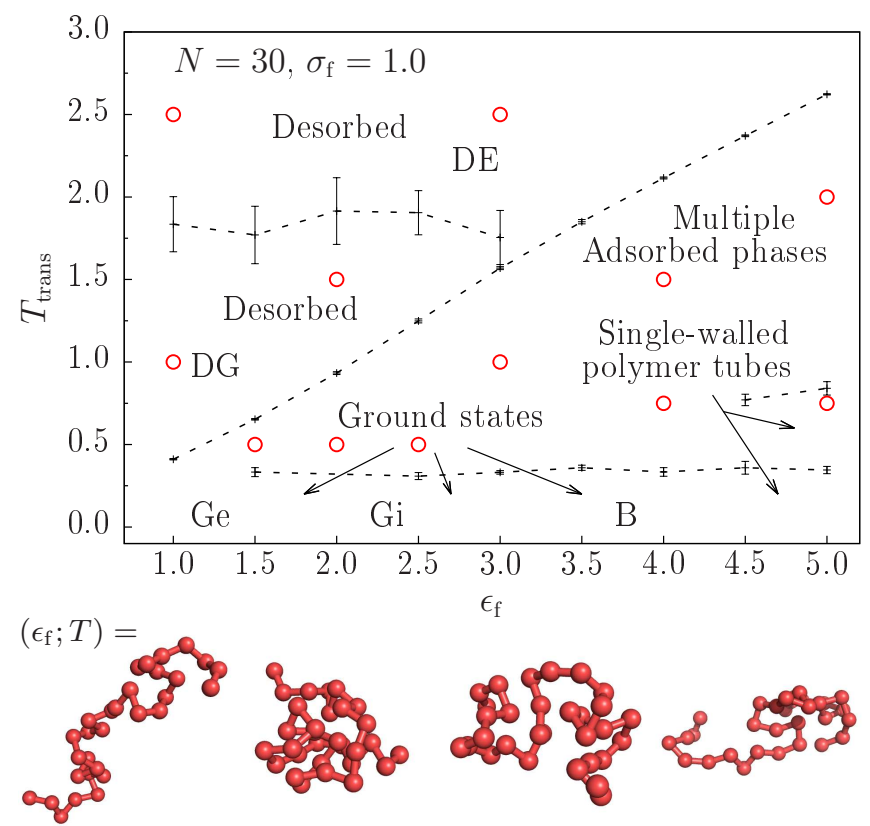

$(1.0 ; 2.5)$
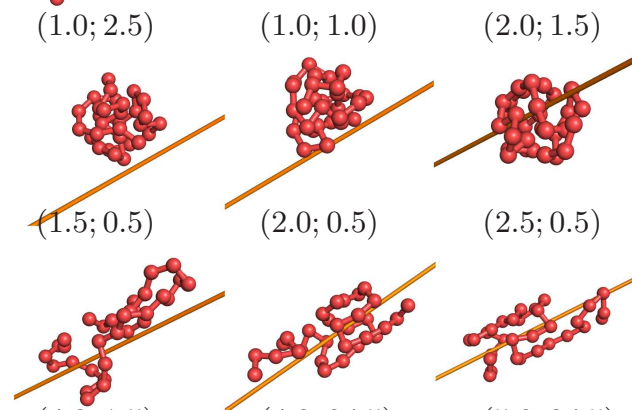

$(4.0 ; 1.5)$

$(2.5 ; 0.5)$

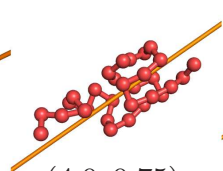

$(4.0 ; 0.75)$

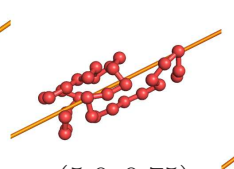

$(5.0 ; 0.75)$

FIG. 5. Example of the structural phase diagram and representative conformations in the different conformational phases for $N=30$ at wire thickness $\sigma_{\mathrm{f}}=1.0$. Transition temperatures $T_{\text {trans }}$ were identified from peaks in the specific heat. The low-energy "clamshell" phase $\mathrm{C}$ does not exist for $\sigma_{\mathrm{f}}=1.0$.

\section{THE ADSORPTION TRANSITION FROM THE MICROCANONICAL PERSPECTIVE}

The canonical statistical analysis of different response quantities, including the specific heat, as functions of the canonical temperature is typically ambiguous. Although signals such as extremal points and inflection points are helpful indicators of thermal activity, they do not allow for a unique identification of transition points [4, 49, 59]. Figure 6] shows the adsorption peaks in the heat-capacity curves for various $\varepsilon_{\mathrm{f}}$ values and wire thickness $\sigma_{\mathrm{f}}=1.0$ for a 30-mer and a 100-mer.

An alternative method that has proven to be extremely useful is based on the fact that temperature can also be defined as a system property. This method is called microcanonical inflection-point analysis [47] and rests upon the shoulders of microcanonical statistical analysis [60].

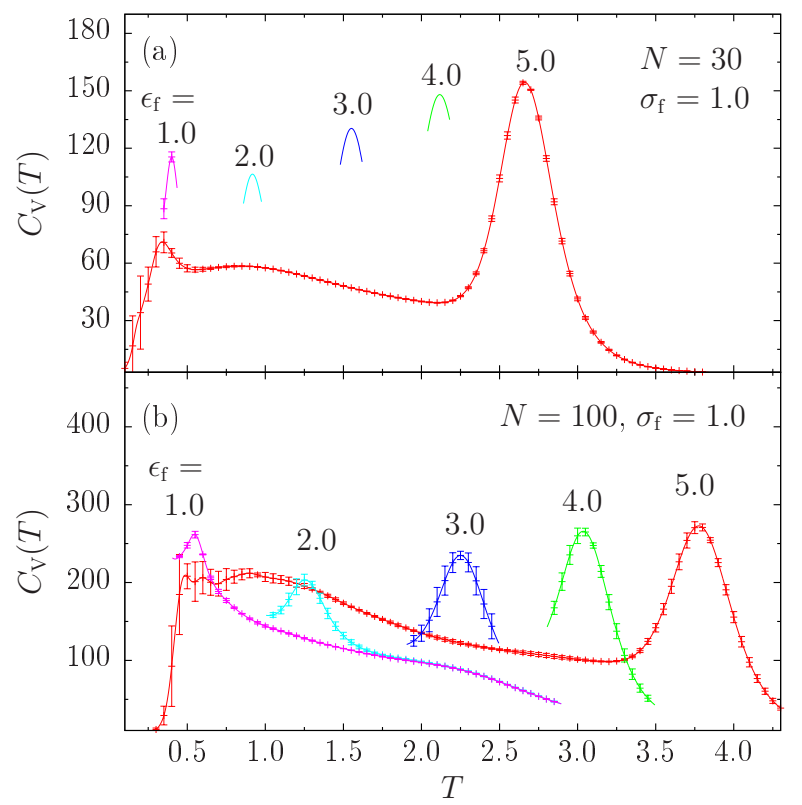

FIG. 6. Adsorption transition signals in the heat-capacity curves of (a) a 30-mer and (b) a 100-mer for different values of the wire attraction strength $\sigma_{\mathrm{f}}$ and fixed effective thickness $\sigma_{\mathrm{f}}=1.0$. For visualization purposes, only the adsorption peaks are shown for $\epsilon_{\mathrm{f}}<5.0$.

The central quantity is the density of states $g\left(E^{\prime}\right)=$ $\int \mathcal{D} X \delta\left(E^{\prime}-E(\mathbf{X})\right)$, which sets entropy and energy into relation with each other: $S(E)=k_{\mathrm{B}} \ln g(E)$. It is easy to show that microcanonical equilibrium between two systems is achieved if $\partial S_{1}\left(E_{1}\right) / \partial E_{1}=\partial S_{2}\left(E_{2}\right) / \partial E_{2}$. With this, the inverse microcanonical temperature can be defined as $\beta(E)=\partial S(E) / \partial E$. Figures 7(a) and (b) show for the 30-mer the microcanonical entropy and inverse temperature curves, $S(E)$ and $\beta(E)$, respectively, for wire material parameters $\varepsilon_{\mathrm{f}}=1.0,1.5,2.0, \ldots, 5.0$ and $\sigma_{\mathrm{f}}=1.0$. For comparison, the same plots are shown in Fig. 8 for a 100-mer.

The $\beta(E)$ curves in Figs. $7(b)$ and $8(b)$ exhibit a characteristic behavior that help locate transition points uniquely. Specific monotonic properties can even be used to introduce a systematic classification scheme. In microcanonical inflection-point analysis [47], the transition points are defined by means of the derivative $\gamma(E)=d \beta(E) / d E$ in the following way. If $\gamma(E)$ exhibits a local maximum and the extremal value is positive, $\gamma_{\max }\left(E_{\mathrm{tr}}\right)>0$, then the associated inflection point of the microcanonical temperature at $E_{\mathrm{tr}}$ must lie in a low-sensitivity region, in which an energetic increase has a minimal effect on the change of temperature within a certain energetic range. For a finite system, this entails the "backbending" of the temperature curve, which levels out in the thermodynamic limit and renders the Maxwell construction exact. The energy that spans the transition range is associated with a nonzero latent heat $\Delta Q>0$, which is why this transition is referred to as a first-order transition. 


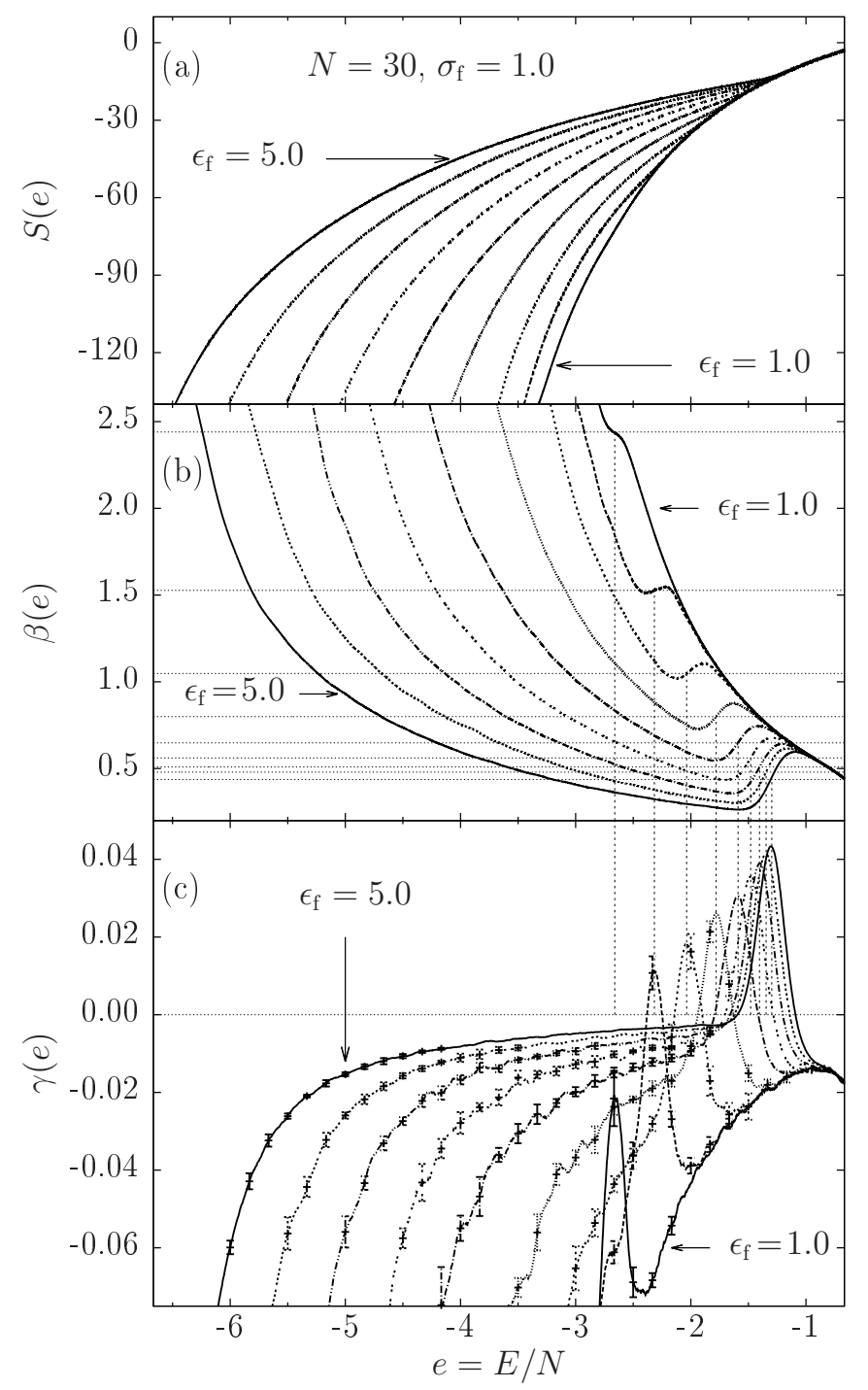

FIG. 7. Microcanonical analysis of structural transitions of the polymer with $N=30$ monomers interacting with wires of effective thickness $\sigma_{\mathrm{f}}=1$ and attraction strengths $\epsilon_{\mathrm{f}}=$ $1.0,1.5,2.0, \ldots, 5.0$. (a) Microcanonical entropy $S(e)$, (b) inverse microcanonical temperature $\beta(e)=(1 / N)(d S(e) / d e)$, and (c) inflection-point indicator $\gamma(e)=(1 / N)(d \beta(e) / d e)$.

Consequently, a negative local maximum $\gamma_{\max }\left(E_{\mathrm{tr}}\right)<$ 0 , which also signals a low-sensitivity region of the $\beta(E)$ curve, marks a second-order transition, because it corresponds to a transition point in energy space and, therefore, $\Delta Q=0$. Note that according to these definitions the order of transition depends on the system size. It is not unusual that a transition with first-order signature in a finite system turns into a continuous, second-order phase transition in the thermodynamic limit. Not surprisingly, the order of a transition is also sensitive to changes of system (or model) parameters.

Most strikingly, as can be seen in Figs. 7(b) and (c) for the 30-mer, the adsorption transition that we classify as a second-order transition at $\varepsilon_{\mathrm{f}}=1.0$, turns into first order

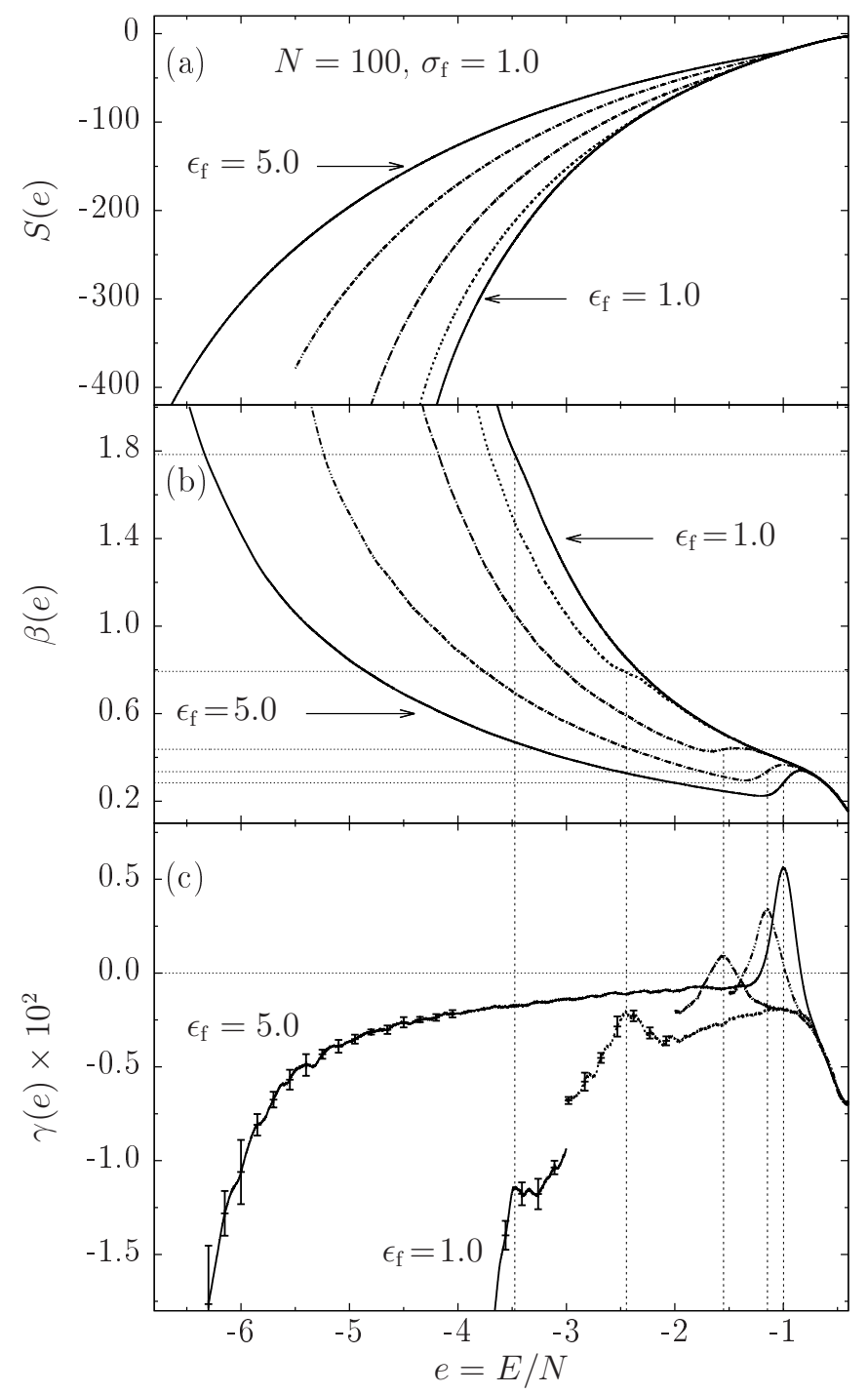

FIG. 8. Same as Fig. 7 but for $N=100$ and attraction strengths $\epsilon_{\mathrm{f}}=1.0,2.0, \ldots, 5.0$.

for larger attraction strengths. The first-order characteristic becomes stronger with increasing $\varepsilon_{\mathrm{f}}$ values. The horizontal lines in Fig. 7(b) represent the (inverse) transition temperatures identified from the corresponding inflection points. They coincide very nicely with the peak values of the specific heat curves shown in Fig. 6. The scenario is similar for the 100-mer. The inverse microcanonical temperatures, plotted in Fig. 8(b) for various $\varepsilon_{\mathrm{f}}$ values, exhibit features of a second-order transition for $\varepsilon_{\mathrm{f}}<3.0$, before turning to first order at higher $\varepsilon_{\mathrm{f}}$ values. This is very similar to the previously discussed case of polymer adsorption at flat substrates 22].

The reason for the increasing entropic suppression of states in the phase separation regime is that less states are available for each energetic state in the linearly increasing energy interval (the energy scale grows like $\left.E \propto \varepsilon_{\mathrm{f}}\right)$. Since the desorption phase is almost entirely governed by translational entropy (in a constant energy 
interval above the adsorption transition), and the energetically controlled adsorption states are shifted to lower energies (below the adsorption transition) with $\varepsilon_{\mathrm{f}}$ increasing, the energetic space in between is entropically "emptied" (note that the total number of states is independent of $\varepsilon_{\mathrm{f}}$ ). For the same reason, the slope of the double-tangents (Gibbs construction) at the respective transition points decreases for larger values of $\varepsilon_{\mathrm{f}}$, which effectively shifts the adsorption transition toward higher temperatures, which is consistent with the canonical analysis of Fig. 6. Since the energy scale of adsorption is linear, the adsorption transition temperature scales almost linearly as well over a large range of the adsorption strength parameter, $T_{\text {ads }} \sim \varepsilon_{\mathrm{f}}$, which is a common feature of all minimally (additively) coupled adsorption models [15, 18, 22].

The increased $\varepsilon_{\mathrm{f}}$ range of second-order behavior of the 100-mer, compared to the 30-mer, is an empirical indication that the adsorption transition of a polymer at a linelike substrate is a second-order phase transition in the thermodynamic limit. This is consistent with the general assumption that adsorption transitions of infinitely large systems are continuous [61]. This means that the width of the phase-separation regime, which is nonzero in the finite system, shrinks with increasing number of monomers and the per-particle latent heat diminishes for $N \rightarrow \infty$ [22].

\section{SUMMARY}

We have investigated the thermodynamic behavior of structural phases for a hybrid model of a polymer interacting with stringlike substrate that resembles a nanowire. This study extends the previous study of groundstate properties of the same system [43] by analyzing the influence of thermal fluctuations upon the adsorption transition and the formation of compact adsorption phases. Results were obtained by means of advanced generalized-ensemble Monte Carlo simulations. We identified all major structural thermodynamic phases from indications of enhanced thermal activity in the specific heat curves and constructed the structural hyperphase dia- gram, parametrized by temperature and string attraction strength. As expected, for increasing attraction strength, the low-temperature structures change from compact crystalline shapes that attach to the string or include it to barrellike conformations that resemble single-walled nanotubes. The transition into less ordered adsorption phases turns out to be independent of the adsorption strength, i.e., the transition temperature is virtually constant. In contrast, the adsorption/desorption transition temperature scales linearly with the substrate attraction strength. A microcanonical inflection-point analysis revealed that the adsorption transition possesses an increasingly strong first-order characteristic, the larger the attraction strength is. However, it is important to note that this first-order behavior is a finite-system feature that is supposed to disappear in the thermodynamic limit. It is only due to an effective, relative suppression of microstates in the phase-separation transition region, which is a finite-size effect. This does not contradict the common view that the adsorption transition is a secondorder phase transition in the thermodynamic limit.

The results presented in this paper provide general background knowledge needed for systematic approaches to the technological design of functional materials used for the transport of molecules (based on polymer tubes) and also give insight into binding options of biomacromolecules at nanofibers (such as actin filaments) in cells.

\section{ACKNOWLEDGMENTS}

This work has been supported partially by the NSF under Grant No. DMR-1207437, by CNPq (National Council for Scientific and Technological Development, Brazil) under Grant No. 402091/2012-4, and by the DFG (German Research Foundation) under Grant No. SFB/TRR 102 (Project B04). The authors also acknowledge support by the Jülich/Aachen/Haifa Umbrella program under Grants No. SIM6 and No. HPC_2. Computer time was provided by the Forschungszentrum Jülich under Project No. jiff39 and No. jiff43, and by the Georgia Advanced Computing Resource Center (GACRC) at the University of Georgia. Assigned: LA-UR-14-27917.
[1] S. R. Whaley, D. S. English, E. L. Hu, P. F. Barbara, A. M. Belcher, Nature 405, 665 (2000).

[2] J. J. Gray, Curr. Opin. Struct. Biol. 14, 110 (2004).

[3] M. Bachmann, K. Goede, A. G. Beck-Sickinger, M. Grundmann, A. Irbäck, and W. Janke, Angew. Chem. Int. Ed. 49, 9530 (2010)

[4] M. Bachmann, Thermodynamics and Statistical Mechanics of Macromolecular Systems, (Cambridge University Press, Cambridge, 2014).

[5] T. Vogel, M. Bachmann, and W. Janke, Phys. Rev. E 76, 061803 (2007).

[6] S. Schnabel, T. Vogel, M. Bachmann, and W. Janke,
Chem. Phys. Lett. 476, 201 (2009).

[7] S. Schnabel, M. Bachmann, and W. Janke, J. Chem. Phys. 131, 124904 (2009).

[8] T. Bogner, A. Degenhard, and F. Schmid, Phys. Rev. Lett. 93, 268108 (2004).

[9] A. Swetnam and M. P. Allen, Phys. Rev. E 85, 062901 (2012).

[10] M. Möddel, W. Janke, and M. Bachmann, Phys. Rev. Lett. 112, 148303 (2014).

[11] T. Vrbová and S. G. Whittington, J. Phys. A 29, 6253 (1996); J. Phys. A 31, 3989 (1998); T. Vrbová and K. Procházka, J. Phys. A 32, 5469 (1999). 
[12] Y. Singh, D. Giri, and S. Kumar, J. Phys. A 34, L67 (2001); R. Rajesh, D. Dhar, D. Giri, S. Kumar, and Y. Singh, Phys. Rev. E 65, 056124 (2002).

[13] M. S. Causo, J. Chem. Phys. 117, 6789 (2002).

[14] J. Krawczyk, T. Prellberg, A. L. Owczarek, and A. Rechnitzer, Europhys. Lett. 70, 726 (2005).

[15] M. Bachmann and W. Janke, Phys. Rev. Lett. 95, 058102 (2005); Phys. Rev. E 73,041802 (2006); Phys. Rev. E 73, 020901(R) (2006).

[16] N. Källrot and P. Linse, Macromolecules 40, 4669 (2007).

[17] J. Luettmer-Strathmann, F. Rampf, W. Paul, and K. Binder, J. Chem. Phys. 128, 064903 (2008).

[18] M. Möddel, M. Bachmann, and W. Janke, J. Phys. Chem. B 113, 3314 (2009).

[19] L. Wang, T. Chen, X. Lin, Y. Liu, H. Liang, J. Chem. Phys. 131, 244902 (2009).

[20] A. D. Swetnam and M. P. Allen, Phys. Chem. Chem. Phys. 11, 2046 (2009).

[21] V. A. Ivanov, J. A. Martemyanova, M. Müller, W. Paul, and K. Binder, J. Phys. Chem. B 113, 3653 (2009).

[22] M. Möddel, W. Janke, and M. Bachmann, Phys. Chem. Chem. Phys. 12, 11548 (2010); Macromolecules 44, 9013 (2011).

[23] Y. W. Li, T. Wüst, and D. P. Landau, Phys. Rev. E 87, 012706 (2013).

[24] H. Popova and A. Milchev, J. Chem. Phys. 127, 194903 (2007); ibid. 129, 215103 (2008).

[25] S. Karalus, W. Janke, and M. Bachmann, Phys. Rev. E 84, 031803 (2011).

[26] A. Milchev and K. Binder, J. Chem. Phys. 117, 6852 (2002).

[27] I. Gurevitch and S. Srebnik, Chem. Phys. Lett. 444, 96 (2007); J. Chem. Phys. 128, 144901 (2008).

[28] J. Gross, T. Vogel, and M. Bachmann, Structural Phases of Adsorption for Flexible Polymers on Nanocylinder Surfaces, to be published (2015).

[29] M. S. Dresselhaus, G. Dresselhaus, and P. Avouris, eds., Carbon Nanotubes: Synthesis, Structure, Properties, and Applications, Topics in Applied Physics, Vol. 80 (Springer, Heidelberg, 2001).

[30] A. Jorio, G. Dresselhaus, and M. S. Dresselhaus, eds., Carbon Nanotubes: Advanced Topics in the Synthesis, Structure, Properties, and Applications, Topics in Applied Physics, Vol. 111 (Springer, Berlin, Heidelberg, 2008).

[31] T. Hasan, Z. Sun, F. Wang, F. Bonaccorso, P. H. Tan, A. G. Rozhin, and A. C. Ferrari, Adv. Mater. 21, 3874 (2009).

[32] M. Gao, L. Dai, and G. G. Wallace, Electroanalysis 15, 1089 (2003).

[33] L. Wang, Y. Liu, W. Li, X. Jiang, Y. Ji, X. Wu, L. Xu, Y. Qiu, K. Zhao, T. Wei, Y. Li, Y. Zhao, and C. Chen, Nano Lett. 11, 772 (2011).
[34] S. H. Brewer, W. R. Glomm, M. C. Johnson, M. K. Knag, and S. Franzen, Langmuir 21, 9303 (2005).

[35] M. Q. Tran, J. T. Cabral, M. S. P. Shaffer, and A. Bismarck, Nano Lett. 8, 2744 (2008).

[36] C. Wei, Nano Lett. 6, 1627 (2006).

[37] S. S. Tallury and M. A. Pasquinelli, J. Phys. Chem. B 114, 4122 (2010); ibid. 9349 (2010).

[38] W. Liu, C.-L. Yang, Y.-T. Zhu, and M.-S. Wang, J. Phys. Chem. C 112, 1803 (2008).

[39] C. Caddeo, C. Melis, L. Colombo, and A. Mattoni, J. Phys. Chem. C 114, 21109 (2010).

[40] E. J. Wallace and M. S. P. Sansom, Nano Lett. 7, 1923 (2007).

[41] E. J. Wallace, R. S. G. D'Rozario, B. M. Sanchez, and M. S. P. Sansom, Nanoscale 2, 967 (2010).

[42] P. Angelikopoulos and H. Bock, Langmuir 26, 899 (2010).

[43] T. Vogel and M. Bachmann, Phys. Rev. Lett. 104, 198302 (2010); Phys. Proc. 4, 161 (2010)

[44] H. D. Wagner, J. Appl. Phys. 67, 1352 (1990).

[45] B. J. Carroll, Langmuir 2, 248 (1986).

[46] R. Glaeser, W. Chiu, and D. Grano, J. Ultrastruct. Res. 66, 235 (1979).

[47] S. Schnabel, D. T. Seaton, D. P. Landau, and M. Bachmann, Phys. Rev. E, 84, 011127 (2011).

[48] F. H. Stillinger, T. Head-Gordon, and C. L. Hirshfeld, Phys. Rev. E 48, 1469 (1993); F. H. Stillinger and T. Head-Gordon, Phys. Rev. E 52, 2872 (1995).

[49] M. Bachmann, H. Arkın, and W. Janke, Phys. Rev. E 71, 031906 (2005).

[50] C. Junghans, M. Bachmann, and W. Janke, Phys. Rev. Lett. 97, 218103 (2006); J. Chem. Phys. 128, 085103 (2008).

[51] T. Vogel and M. Bachmann, Comp. Phys. Comm. 182, 1928 (2011).

[52] B. A. Berg and T. Neuhaus, Phys. Lett. B 267, 249 (1991); Phys. Rev. Lett. 68, 9 (1992).

[53] W. Janke, Physica A 254, 164 (1998).

[54] M. Bachmann, Phys. Scr. 87, 058504 (2013).

[55] F. Wang and D. P. Landau, Phys. Rev. Lett. 86, 2050 (2001); Phys. Rev. E 64, 056101 (2001).

[56] C. Zhou and R. N. Bhatt, Phys. Rev. E 72, 025701(R) (2005).

[57] T. Vogel, T. Mutat, J. Adler, and M. Bachmann, Commun. Comp. Phys. 13, 1245 (2013); Phys. Proc. 15, 87 (2011).

[58] For a review, see C. Shen, A. H. Brozena, Y. Wang, Nanoscale 3, 503 (2011).

[59] M. Bachmann and W. Janke, J. Chem. Phys. 120, 6779 (2004).

[60] D. H. E. Gross, Microcanonical Thermodynamics (World Scientific, Singapore, 2001).

[61] E. Eisenriegler, K. Kremer, and K. Binder, J. Chem. Phys. 77, 6296 (1982). 